\title{
Application of COOK ok Cervical Ripening Balloon Combined With Artificial Rupture of Membranes and Oxytocin in Labor Induction for Full-term Pregnancy
}

\section{Ying Dong}

Shanghai PuTuo District Center Hospital

\section{Chuyu Li}

Shanghai Red House Obstetrics and Gynecology Hospital: Obstetrics and Gynecology Hospital of Fudan University

\section{Xin Zhao}

Shanghai PuTuo District Center Hospital

\section{Lin Zhang}

Shanghai PuTuo District Center Hospital

\section{Xiaojun Jia}

Shanghai PuTuo District Center Hospital

\section{Zhongxing Fu}

Ningguo Bio-leader biotechnology

\section{Yan Du}

Obstetrics and Gynecology Hospital of Fudan University

\section{Ling Wang ( $\sim$ Dr.wangling@fudan.edu.cn )}

Obstetrics and Gynecology Hospital of Fudan University https://orcid.org/0000-0003-4905-9903

\section{Research}

Keywords: COOK balloon, artificial rupture of membranes, oxytocin, labor induction, full-term pregnancy

Posted Date: October 28th, 2020

DOI: https://doi.org/10.21203/rs.3.rs-96849/v1

License: (c) (i) This work is licensed under a Creative Commons Attribution 4.0 International License. Read Full License 


\section{Abstract}

Background The use of COOK balloon in the process of induction of labor is gradually promoted. This study was conducted to investigate the safety and efficacy of COOK double balloon dilation for preinduction cervical maturation and induction of labor.

Methods A total of 343 pregnant women with full-term pregnancy in Shanghai Putuo Maternity \& Infant Health Hospital from January 1st to September 30th of 2016 were enrolled. Of all the pregnant women, 166 had labor induction, which included the use of a COOK balloon, the implementation of artificial rupture of membranes (AROM) and oxytocin intravenous (IV) drip (COOK group). The other 177 pregnant women with spontaneous rupture of membranes (SROM) and mature cervix only administrated with oxytocin IV drip to promote regular uterus contraction (oxytocin group). Maternal adverse reactions, mode of delivery and delivery outcomes of two groups were subsequently compared between the two groups.

Results There were significant differences of maternal age ( $28.29 \pm 3.34$ vs. $29.25 \pm 3.62$ years, $P=0.02)$, gestational age $(283.49 \pm 4.53$ vs. $274.71 \pm 7.04$ years, $P<0.001)$ and birth weight $(3435.27 \pm 340.29$ vs . $3354.63 \pm 387.96 \mathrm{~g}, \mathrm{P}=0.02$ ) between the COOK group and the oxytocin group. There were no significant differences in terms of gravidity, parity, mode of delivery, analgesia, 1 min and 5 min Apgar score, labor time, postpartum hemorrhage, and adverse events.

Conclusion Use of a COOK balloon may help full-term pregnancy women with immature cervix obtain the same pregnancy results as those with mature cervix, without extra occurrence of adverse events.

Trial registration This is an observational study and no registration is required.

\section{Plain English Summary}

When pregnancy complications occur, cesarean section (CS) is a method to save the lives of women and fetus. However, the current global rate of CS is not reasonable. Promoting cervical ripening can reduce the use of CS. This prospective study evaluated the role of COOK balloon in promoting cervical ripening and the incidence of adverse events.

Among the 343 full-term pregnant women admitted to Shanghai Putuo Maternity \& Infant Health Hospital, 166 patients were treated with COOK balloon, artificial rupture of membranes (AROM) and intravenous (IV) oxytocin drip (COOK group). The other 177 pregnant women with spontaneous membrane rupture (SROM) and mature cervix only used oxytocin IV infusion to promote regular uterine contractions (oxytocin group). Subsequently, the adverse maternal reactions, delivery methods and relevant results were compared between the two groups.

There were significant differences in maternal age, gestational age and birth weight between the COOK group and the oxytocin group. There were no significant differences in terms of gravidity, parity, mode of delivery, analgesia, 1 min and 5 min Apgar score, labor time, postpartum hemorrhage, and adverse events. 
In conclusion, using COOK balloons can help full-term pregnant women with immature cervix to obtain the same pregnancy results as pregnant women with mature cervix without additional adverse events.

\section{Background}

Cesarean section (CS) is a life-saving method for women and newborns when complications such as prenatal haemorrhage, fetal distress, abnormal fetal performance, and hypertension occur. In the past 30 years, the global rate of CS has increased by $10-15 \%$, which is much higher than the optimal rate (1). This increase is due to non-medically indicated CS in many middle- and high-income countries (1). Because of poor access to medically indicated CS, CS still accounts for less than $10 \%$ of births in the total population in many low- and middle-income countries (1). As for China, the CS rate has changed from $45.3 \%$ in 2012 to $41.1 \%$ in 2016 (2). With the implementation of China's two-child policy, the above result suggests that caesarean sections declined by 18\% from 2012 to 2016 (2). However, there were vast regional differences, the CS rate in Northeast Jilin Province was as high as 62\%, while 4\% in Tibet in 2014 (3). There is yet no consensus on the optimal frequency of CS at the population level (4). However, it is believed that the current global frequency of CS is medically unreasonably high (5). In China, almost all women deliver at hospitals nowadays and many choose CS, while a high proportion of those operations are considered as having no medical indications (6-9). It is well accepted that CS can provide life-saving interventions for infants in certain conditions such as cord prolapse, separation of the placenta before birth, or the presence of active herpes lesions especially with primary infections (10). As for mothers, CS can protect their health and lives from adverse events including haemorrhage, severe toxemia, severe coexisting medical diseases, or the absolute contraction of the pelvis with a large infant (10). However, there is no evidence that CS is beneficial for infants and women without proper medical indications (1). Just as any surgery, there are short-term and long-term risks, and CS is no exception. CS is associated with increased risk of abnormal placenta, stillbirth, uterine rupture, ectopic pregnancy and premature delivery, and these risks increase in a dose-response manner (11). Accumulating evidences have shown that infants born by CS have different exposures to hormonal, physical, bacterial and medical interventions (11). These interventions can subtly alter the physiology of newborns (11). Short-term risks of CS include changes in immune development; increased possibility of allergies, atopy and asthma; and decreased intestinal microbiome diversity (11). The correlation between CS and the increased incidence of late childhood obesity as well as asthma has been frequently reported (11). In addition, the overused CS resources may be allocated to other medically necessary objectives (12). Therefore, the control of CS is an important issue in China.

Induction of labor (IOL) is the stimulation of uterine contractions during pregnancy before labor initiation to achieve a vaginal delivery. One of the most important factors for predicting the possibility of a successful IOL is the softness and dilation of the cervix (cervical maturity) (13). Conventional labor induction methods include oxytocin intravenous (IV) drip and artificial rupture of membranes (AROM). A small dose of oxytocin IV drip is a safe and common method for IOL. Nevertheless, when the cervix is immature, the effect of IOL may be unsatisfactory (13). Oxytocin acts through the combination with oxytocin receptors. Since few receptors locate in the cervix, direct effects of oxytocin on the cervix 
including promoting cervical maturation are weak (14). AROM is an operation of artificially breaking the fetal membrane, which will later stimulate the release of endogenous prostaglandins (PGE) and oxytocin, and induce contractions of uterus. This operation should be implemented in patients with ideal cervical conditions (13).

Promoting cervical maturation can be interpreted as promoting cervical softening, thinning and expansion; reducing IOL failure rate; and shortening the time from IOL to delivery. If patients with clear IOL indications have an immature cervix, methods of promoting cervical maturation should be adopted. For nulliparous women with an immature cervix, once IOL is implemented, the risk of CS will double (13). At present, the most commonly used method for assessing cervical maturity is the Bishop score. Bishop score $\geq 6$ indicates mature cervix, with a higher score indicating higher success rate of IOL (13). Bishop score $<6$ indicates immature cervical that requires cervical maturation promotion (13). Commonly used methods to promote cervical maturation include PGE (dinoprostone and misoprostol) administration and mechanical methods such as Foley catheters and Cook cervical ripening balloons. Mechanical methods can only be used when the membrane is complete (13). Many studies have compared IOL effects among PGE and different types of balloons. Researchers have reported that IOL duration of PGE is shorter than mechanical methods, but tachysystole and other side effects occur more frequently under PGE administration (15). In contrast, the risk of hyper-stimulation of mechanical methods is lower than that of PGE, while the risks of CS and infection are similar $(16,17)$. Other advantages of mechanical methods include stable at room temperature and relatively easy to insert (17). For the above reasons, mechanical methods have been gradually recommended by the American Congress of Obstetricians and Gynaecologists (18), World Health Organization (19) and Canada (20) IOL guidelines.

However, to the best of our knowledge, so far, no studies have compared spontaneous rupture of membrane (SROM) pregnant women with a mature cervix to pregnant women in need of cervical maturation promotion. The current study compared the CS rate and occurrence rates of related adverse events between pregnant women with an immature cervix used COOK balloons to promote cervical maturation and SROM pregnant women with a mature cervix. This study aimed to provide more evidence for the use of COOK balloons, so that pregnant women with an immature cervix can obtain similar results to pregnant women with a mature cervix.

\section{Methods}

\section{Study design}

This prospective study evaluated the effect of COOK balloons, and investigated whether pregnant women with an immature cervix can obtain same results as SROM pregnant women with a mature cervix.

\section{Subjects}

Three hundred and forty-three pregnant women with full-term pregnancy in Shanghai Putuo Maternity \& Infant Health Hospital from January 1st to September 30th of 2016 were enrolled in the study. All women 
had an investigation of detailed personal, medical and obstetric histories; general, abdominal and vaginal examinations; an abdominal ultrasonography and a cardiotocography (CTG) examination. Inclusion criteria were: full-term pregnancy, singleton pregnancy, cephalic fetal presentation and wish to have a vaginal delivery. Exclusion criteria were: histories of uterine body surgery, previous CS deliveries, macrosomia (expected neonatal weight $>4 \mathrm{~kg}$ ), non-reassuring fetal conditions, placenta previa and malpresentations. Then those women were allocated to two groups according to cervix and membrane conditions. Pregnant women with an intact membrane and an immature cervix (Bishop score $\leq 4$ ) were allocated to the COOK group, while SROM pregnant women with a mature cervix (Bishop score $\geq 6$ ) were allocated to the oxytocin group. The COOK group received COOK balloon placement combined with AROM and oxytocin IV drip, and the oxytocin group received only oxytocin IV drip. The study complied with the Declaration of Helsinki, Ethical Principles for Medical Research Involving Human Subjects, and was approved by the ethical committee in Shanghai Putuo Maternity \& Infant Health Hospital. Informed consent was obtained from each participant.

\section{Methods}

In the COOK group, a double-balloon catheter was inserted according to manufacturer's instructions. The catheter was then taped to the inner thigh in order to reduce discomfort. Preparations for catheter insertion were as follows. After performing an abdominal ultrasound examination, the woman was placed in the lithotomy position. A large vaginal speculum was inserted into the vagina to obtain access to the cervix. The cervix was then washed with a cleaning solution before insertion of the catheter. Later, the catheter was inserted into the cervix until both balloons entered the cervical canal. The uterine balloon was inflated with $40 \mathrm{~mL}$ normal saline (NS) by a standard $20 \mathrm{~mL}$ syringe, and the injection was through the red Check-Flo valve marked ' $U$ '. After the inflation of the uterine balloon, the device was pulled back until the uterine balloon hit against the internal cervical os. The vaginal balloon became visible outside the external cervical os. The vaginal balloon was inflated with $20 \mathrm{~mL}$ NS by a standard $20 \mathrm{~mL}$ syringe, and the injection was through the green Check-Flo valve marked ' $V$ '. Once the balloons were situated on each side of the cervix and the device was fixed in place, the speculum was removed. More NS was injected into each balloon in turn, at $20 \mathrm{~mL}$ increments, until each balloon containing $80 \mathrm{~mL}$ (maximum) NS. If needed, the proximal end of the catheter could be taped to the woman's thigh (21). Removal of the balloon was executed at approximately 12 hours after insertion if spontaneous expulsion did not occur. AROM and oxytocin IV drip were commenced if the labor did not start spontaneously after removal or spontaneous expulsion of the catheter. Oxytocin IV drip was administrated according to the WHO recommendations for induction of labor. Continuous electronic fetal monitoring was used throughout the labor process. Abnormalities during labor were diagnosed and managed according to the recommendations from the American College of Obstetricians and Gynaecologists guideline. The following items were recorded: mode of delivery, use of analgesia, birth weight, Apgar score at $1 \mathrm{~min}$ and 5 min, labor time, amount of postpartum haemorrhage, and numbers of adverse events including hyperstimulation, postpartum urinary retention, puerperal infection, cord prolapse, placental abruption and obstetric laceration of cervix. 
In the oxytocin group, the pregnant women were administrated with oxytocin IV drip 2 hours after SROM. The usage and dosage of oxytocin were based on the WHO recommendations for IOL. Continuous electronic fetal monitoring was used throughout the labor process. The following items were recorded: mode of delivery, use of analgesia, birth weight, Apgar score at $1 \mathrm{~min}$ and $5 \mathrm{~min}$, labor time, amount of postpartum haemorrhage, and numbers of adverse events including hyperstimulation, postpartum urinary retention, puerperal infection, cord prolapse, placental abruption and obstetric laceration of cervix.

All data were quality checked and entered twice into a secure electronic database. Paper and electronic data were stored at a designated research office in the hospital, and can only be accessed by research staff. Paper data were securely stored in a locked filling cabinet, and electronic data were stored in a password-protected computer. Follow-up information was linked to the basic clinicopathological database through unique ID numbers of the participants. All identifiable data were removed before analyses to protect subjects' privacy.

\section{Statistical analysis}

Number and percentage were calculated for categorical variables. Chi-square test or Fisher's exact test were used to determine the differences of categorical variables. Mean and standard deviation (SD) or median and range were calculated for continuous variables. Continuous variables were tested for normality and equality of variances between groups. Student's t test was used to compare continuous variables that were normally distributed, and nonparametric methods were used to compare not normally distributed variables. All above analyses were two-sided and performed using SPSS version 22 for Windows. A P value of $<0.05$ was considered statistically significant.

\section{Results}

\section{Population characteristics}

A total of 343 participants were enrolled in this study. All were full-term ( 37 to $41+6$ weeks) pregnant women and met the inclusion criteria. Results of comparisons between the COOK group and the oxytocin group are shown in Table 1. The maternal age of the COOK group was significantly younger than that of the oxytocin group ( $28.29 \pm 3.34$ vs. $29.25 \pm 3.62$ years, $P=0.02)$. There were also statistically significant differences of gestational age at induction ( $283.49 \pm 4.53$ vs. $274.71 \pm 7.04$ days, $P<0.001)$. Regarding gravidity $(G)$ and parity $(P)$ histories, there were no statistically significant differences between the two groups ( $P>0.05$ for both). 
Table 1

Baseline characteristics of the COOK group versus the oxytocin group

\begin{tabular}{|llll|}
\hline & COOK group $(\mathbf{n = 1 6 6 )}$ & Oxytocin group $(\mathbf{n = 1 7 7 )}$ & $P$ value \\
\hline Age (years) & $28.29 \pm 3.34$ & $29.25 \pm 3.62$ & 0.02 \\
\hline Gestational age at induction (days) & $283.49 \pm 4.53$ & $274.71 \pm 7.04$ & $<0.001$ \\
\hline Gravidity (median) & $1(1-10)$ & $1(1-7)$ & 0.95 \\
\hline Parity (n, \%) & & & 0.46 \\
\hline 0 & $153(92.2 \%)$ & $158(89.3 \%)$ & \\
\hline 1 & $13(7.8 \%)$ & $18(10.2 \%)$ & \\
\hline 2 & $0(0 \%)$ & $1(0.5 \%)$ & \\
\hline
\end{tabular}

\section{Delivery outcomes of COOK group versus oxytocin group}

Table 2 shows outcomes of both mothers and infants in the COOK group and the oxytocin group. Birth weight of the COOK group was significantly heavier than that of the oxytocin group (3435.27 \pm 340.29 vs. $3354.63 \pm 387.96 \mathrm{~g}, P=0.02$ ). As for mode of delivery, use of analgesia, $1 \mathrm{~min}$ and $5 \mathrm{~min}$ Apgar score, there were no statistically significant differences between the two groups $(P>0.05$ for all). Then we restricted the analysis to those without CS (30 received CS in the COOK group and 37 received CS in the oxytocin group) and compared labor time. There were no significant differences between the two groups in terms of first labor time, second labor time and total labor time $(P>0.05$ for all). 
Table 2

Delivery outcomes of the COOK group versus the oxytocin group

\begin{tabular}{|c|c|c|c|}
\hline & $\begin{array}{l}\text { COOK group }(n= \\
166)\end{array}$ & $\begin{array}{l}\text { Oxytocin group }(n= \\
177)\end{array}$ & $\begin{array}{l}P \\
\text { value }\end{array}$ \\
\hline Mode of delivery $(n, \%)$ & & & 0.76 \\
\hline Vaginal delivery & $128(77.1 \%)$ & $133(75.1 \%)$ & \\
\hline Forceps delivery & $8(4.8 \%)$ & $7(4.0 \%)$ & \\
\hline CS & $30(18.1 \%)$ & $37(20.9 \%)$ & \\
\hline Analgesia (n, \%) & & & 0.27 \\
\hline Yes & $118(71.1 \%)$ & $116(65.5 \%)$ & \\
\hline No & $48(28.9 \%)$ & $61(34.5 \%)$ & \\
\hline Birth weight (g) & $3435.27 \pm 340.29$ & $3354.63 \pm 387.96$ & 0.02 \\
\hline \multicolumn{4}{|l|}{ Apgar score } \\
\hline $1 \mathrm{~min}$ & $9.87 \pm 0.63$ & $9.94 \pm 0.44$ & 0.22 \\
\hline $5 \mathrm{~min}$ & $9.98 \pm 0.24$ & $9.99 \pm 0.08$ & 0.52 \\
\hline $\begin{array}{l}\text { Labor time (min) (CS patients } \\
\text { excluded) }\end{array}$ & $\begin{array}{l}\text { COOK group }(n= \\
136)\end{array}$ & $\begin{array}{l}\text { Oxytocin group }(n= \\
140)\end{array}$ & \\
\hline First labor time & $239.49 \pm 106.31$ & $226.68 \pm 85.02$ & 0.42 \\
\hline Second labor time & $46.61 \pm 32.72$ & $52.47 \pm 35.65$ & 0.14 \\
\hline Total labor time & $291.58 \pm 116.94$ & $284.64 \pm 102.45$ & 0.90 \\
\hline
\end{tabular}

\section{Adverse events of COOK group versus oxytocin group}

Table 3 displays adverse events of the COOK group versus the oxytocin group. Neither groups had serious adverse events such as cord prolapse and placental abruption. There was no statistically significant difference in amount of postpartum haemorrhage ( $303.13 \pm 72.34$ vs. $303.87 \pm 89.89 \mathrm{~mL}, P>0.05)$. A total of 7 adverse events occurred in the COOK group: 5 cases of postpartum urinary retention, and 2 cases of puerperal infection. A total of 9 adverse events occurred in the oxytocin group: 4 cases of postpartum urinary retention, 3 cases of puerperal infection, 1 case of hyperstimulation, and 1 case of obstetric laceration of cervix. There were no statistically significant differences of the adverse event rate between the two groups $(P>0.05)$. 
Table 3

Labor indexes and neonatal outcomes of the COOK group versus the oxytocin group

\begin{tabular}{|llll|}
\hline & COOK group $(\mathrm{n}=166)$ & Oxytocin group $(\mathrm{n}=177)$ & $P$ value \\
\hline Postpartum haemorrhage $(\mathrm{mL})$ & $303.13 \pm 72.34$ & $303.87 \pm 89.89$ & 0.65 \\
\hline Total adverse events $(\mathrm{n}, \%)$ & $7(4.2 \%)$ & $9(5.2 \%)$ & 0.14 \\
\hline Hyperstimulation $(\mathrm{n}, \%)$ & $0(0.0 \%)$ & $1(0.6 \%)$ & 0.94 \\
\hline Postpartum urinary retention $(\mathrm{n}, \%)$ & $5(3.0 \%)$ & $4(2.3 \%)$ & 0.19 \\
\hline Puerperal infection $(\mathrm{n}, \%)$ & $2(1.2 \%)$ & $3(1.7 \%)$ & 0.14 \\
\hline Obstetric laceration of cervix $(\mathrm{n}, \%)$ & $0(0.0 \%)$ & $1(0.6 \%)$ & 0.94 \\
\hline
\end{tabular}

\section{Discussion}

In this study, we compared delivery results and adverse events between the COOK group and the oxytocin group. We found that pregnant women receiving COOK balloons had almost same delivery results and adverse events as SROM pregnant women with a mature cervix. As for baseline characteristics, average age of the COOK group was significantly younger than the oxytocin group. In addition, gestational age of the COOK group was older than that of the oxytocin group. Previous studies have reported that women with advanced maternal age were more likely to have a preterm delivery (22), suggesting a younger gestational age. Therefore, older gestational age of the COOK group may be explained by younger maternal age. In addition, a number of studies have shown that there is a direct positive correlation between birth weight and gestational age (23-25). Hence, it is possible that heavier birth weight of the COOK group may be partially explained by older gestational age.

The rate of mode of delivery was almost the same in the COOK group (18.1\%) and the oxytocin group (20.9\%). Since both nulliparous women and multiparous women were included in our study, and combined with inclusion and exclusion criteria, our participants belonged to the $2 \mathrm{a}$ and 4 a groups according to the Robson ten-group classification system. After excluding multiparous women, CS rate was $19.6 \%$ (30 of 153) in the COOK group and $22.8 \%$ (36 of 158) in the oxytocin group, respectively. Wu et.al conducted a survey in six midwifery institutions of Shanghai, and reported that the CS rate of the $2 \mathrm{a}$ group was $14.53 \%$ in 2015 and $2.13 \%$ in 2017 , respectively (26). Our results showed higher CS rate compared with their study, which may have several possible explanations. First, the two-child policy was implemented nationwide from January 1 st of 2016 and our study time-span is from January 1 st to September 30th of 2016. It is highly likely that most of the women in our study became pregnant before the implementation of this policy. Studies from central China have reported that after the implementation of the two-child policy, the CS rate in nulliparous patients have decreased (27). Therefore, two-child policy may be one possible explanation for the low CS rate in 2017. Second, Shanghai Putuo Maternity \& Infant Health Hospital is a specialized grade 2A hospital, and locates in the urban area. In a study by Zhang et.al, they found that the CS rate of the 2A hospital was higher than that of the $3 \mathrm{~A}$ hospital (28), which 
may be related to the fact that secondary hospitals are less capable than tertiary hospitals in prenatal testing, diagnosis and management of maternal and fetal complications, evaluation of surgical indications, and supervision. These reasons lead to the implementation of more CS operations without specified medical indications (28).

Some studies have also compared effects of COOK balloons and oxytocin in promoting ripening of cervixes in pregnant women with full-term singleton pregnancy, cephalic fetal presentation and wish to have vaginal delivery. In the study by Fan et.al, the effects of COOK balloons and oxytocin in pregnant women with immature cervix and complete membrane were compared, and no statistically significant differences of maternal age, gestational age, and Bishop score before the use of balloons and oxytocin were observed between the two groups. They reported that the CS rate was significantly different $(18.75 \%$ vs. $35 \%, P<0.05)$ of the two groups (14). In the study by Han et.al, although there were statistical differences of age and gestational age between the two groups, no statistically significant differences of neonatal weight, cervical Bishop score before labor induction, and labor induction indicators were observed. They also reported that the CS rate was significantly different $(20.4 \%$ vs. $37.7 \%, P<0.05)$ of the two groups (29). The reason we did not find significant differences of CS rates between the two groups might be that patients in the oxytocin group had a mature cervix. One strength of our study is that we evaluated whether the placement of COOK balloons can achieve same results as SROM pregnant women with a mature cervix. In addition, we found similar CS rate (18.1\%) in the COOK group to the studies of Fan et.al (18.75\%) and Han et.al (20.4\%). Therefore, our study provides an argument for the role of COOK balloons in promoting cervical maturation and reducing CS rate.

According to the "Guidelines for Promoting Cervical Maturity and Induction of Labor in Late Pregnancy", the advantages of COOK balloons are low cost, stable at room temperature, and low risk of excessive contractions compared with PGE preparations. Disadvantages of COOK balloons include the possibility of potential infections, premature rupture of membranes, and cervical damage (13). Duro-Gómez reported the cost of misoprostol for IOL was lower than that of dinoprostone or COOK balloons, with similar obstetric and perinatal outcomes (15). Fan et.al reported that the success rate of COOK balloons for cervical ripening $(100 \%)$ was significantly higher than that of oxytocin $(79.38 \%)$, and the vaginal delivery rate was significantly higher in the balloon group $(81.25 \%$ vs. $65 \%, P<0.05)$ as well. In addition, the balloon did not increase maternal complications or adverse infant outcomes, such as umbilical cord prolapses, intrauterine infection, uterine hyperstimulation, and placental abruption. The time span of the first stage of labor in the COOK group was significantly shorter than that in the oxytocin group $(6.1 \pm 1.3$ vs. $10.5 \pm 3.2 \mathrm{~h}, P<0.05)$. Furthermore, there were no statistically significant differences in the Apgar score, weight and postpartum haemorrhage rates between the two groups (14). In Han's study, after removing the COOK cervical dilatation balloon, the Bishop score of the experiment group increased from $2.38 \pm 0.7$ points before placement to $6.15 \pm 0.96$ points after placement. The vaginal delivery rate of the COOK group was significantly higher than that of the oxytocin group $(79.6 \% \mathrm{vs.} 62.3 \%, P=0.005)$. The occurrence rate of chorioamnionitis in the COOK balloon group was significantly higher than that in the oxytocin group $(18.4 \%$ vs. $5.4 \%, P=0.002)$. There were no significant differences in other adverse event rates such as uterine hyperstimulation, rapid birth, neonatal asphyxia, postpartum haemorrhage, cervical 
laceration, or placental abruption (29). In the study by Lim et.al, there was no difference in the pain score between the two groups at the beginning of the IOL. However, thereafter, patients in the COOK group had lower pain scores than patients in the PGE group ( $4.5 \pm 2.3$ vs. $5.6 \pm 2.4, P=0.044)$. Women were equally satisfied with both methods and equally likely to recommend their own methods for IOL (17). In our study, there were no significant differences in adverse events between the two groups. Besides, we found there were no differences in terms of first labor time, second labor time and total labor time between the two groups. While heavier birth weight in the COOK group may be explained by older gestational age. Since the ripening procedure of the cervix in the oxytocin group was a physiological procedure, it is reasonable to conclude that use of Cook balloons may be as effective as the physiological procedure in inducing cervical maturity without extra adverse events.

As for other mechanical methods, Sayed Ahmed reported that compared with Foley catheter, use of Cook balloons would result in greater cervical maturity, which can be reflected as a higher Bishop score after balloon expulsion/removal. The duration from balloon insertion to expulsion and then delivery was significantly shorter when using Foley catheter. There were no differences in pain during insertion, pain after insertion, CS rate, post-partum haemorrhage rate and patient satisfaction (30). In the study of MeiDan et.al, compared with the COOK cervical mature balloon group, the Foley catheter group had a significantly shorter time from balloon insertion to expulsion ( $6.9 \pm 4.2$ vs. $10.1 \pm 4.7 \mathrm{~h}, P=0.001)$ and from insertion to delivery ( $19.6 \pm 11.4$ vs. $23.4 \pm 15.5 \mathrm{~h}, P=0.03)$. There were no significant differences of other outcomes including use of analgesia, episiotomy, operative vaginal delivery, ripening success rate, CS rate, birth weight, 1 min and 5 min Apgar score, pain perception during catheter insertion, hospitalization length and maternal satisfaction (31). Xing et.al reported that single balloon was more effective than double balloons in cervical ripening. The double balloon catheter increased the time from insertion to removal of the catheter and reduced the spontaneous elimination rate of the catheter. All neonatal outcomes in the two groups were comparable (32). Therefore, we may conclude that COOK balloons are more effective than Foley catheters in promoting cervical ripening. The duration from balloon insertion to expulsion and then delivery is significantly shorter when using Foley catheters. Other outcomes as well as CS rate are not different between the two types of catheters.

Mechanical methods mainly apply pressure on the internal os of the cervix, overstretch the lower uterine segment and promote local synthesis and release of endogenous PGE in the cervix, which induces cervical softening and ripening $(13,33)$. Compared with Foley catheters which have a single balloon, double-balloon catheters can apply stable and mild pressure on both external and internal os $(32,34)$, and continuously dilate cervical canals mechanically (29). COOK balloons can open the cervix by 2 to $3 \mathrm{~cm}$ without contractions (14).

There are several strengths of our study. First, to the best of our knowledge, this was the first study to compare CS rate and number of adverse events in pregnant women under the placement of COOK balloons with pregnant women in physiological conditions. In the oxytocin group, oxytocin was only used for promoting regular contractions, since the cervixes of SROM pregnant women were mature. Therefore, our results may suggest that $\mathrm{COOK}$ balloons are able to promote cervix ripening. At the same time, we

Page $11 / 15$ 
also compared occurrences of adverse events in the two groups, and found that there were no significant differences in adverse events, which further confirmed the safety of COOK balloons. However, previous researches have reported that the CS rate correlated positively with maternal age [2], and we cannot rule out the confounding of maternal age on CS rate in the current study. Additionally, Bishop scores before and after the use of balloons were not recorded, therefore the effect of COOK balloons in the change of Bishop scores cannot be directly evaluated.

\section{Conclusion}

To summarize, use of COOK balloons may help pregnant women with immature cervix obtain same pregnancy results as SROM pregnant women with a mature cervix, without extra adverse events.

\section{Abbreviations}

CS

Cesarean Section; IOL:Induction Of Labor; IV:Intravenous; AROM:Artificial Rupture Of Membranes; PGE:Prostaglandins; SROM:Spontaneous Rupture Of Membrane; CTG:Cardiotocography; NS:Normal Saline; SD:Standard Deviation; G:gravidity; P:parity

\section{Declarations}

\section{Ethics approval and consent to participate}

The study was approved by the ethical committee in Shanghai Putuo Maternity \& Infant Health Hospital. Informed consent was obtained from each participant.

\section{Consent for publication}

Not applicable.

\section{Competing interests}

The authors declare that they have no competing interests

\section{Funding}

This work was supported by grants from the National Natural Science Foundation of China (no. 31571196 to Ling Wang and no. 30801502 to Ling Wang), the Program to Guide Medicine ("Yixueyindao") of the Shanghai Municipal Science and Technology Commission (no. 18401902200 to Ling Wang and no. 15401932200 to Ling Wang), the Natural Science Foundation Project of the Shanghai 
Municipal Science and Technology Commission (no. 20ZR1409100 to Ling Wang), the Shanghai Program for Support of Leading Disciplines-Integrative Medicine (no. 20180101 and no. 20150407), the Research Foundation ("CR Sanjiu") of Obstetrics \& Gynecology committee of Chinese Association of Integrated Traditional Chinese and Western Medicine (CR1901FC01 to Ling Wang), the Shanghai Committee of the China Democratic League (no. 02054 to Ling Wang), the FY2008 JSPS Postdoctoral Fellowship for Foreign Researchers (P08471 to Ling Wang), and the Shanghai Pujiang Program (no. 11PJ1401900 to Ling Wang).

\section{Authors' contributions}

YD designed the initial study. All authors participated in the conceptualization and design of the study. YD drafted the initial manuscript. All authors contributed to the development of the selection criteria, the risk of a bias assessment strategy, and data extraction criteria. YD analysed and interpreted the patient data. The manuscript was written by $\mathrm{CL}$ and reviewed by the other co-authors. Altogether the authors read, provided feedback, and permitted the final manuscript before submission. All authors read and approved the final manuscript.

\section{Acknowledgments}

We acknowledge the work of Xuemin Qiu, Lisha Li, Na Zhang, Jing Zhou, Zengshu Huang, Wingting Leung, Xinyao Pan, and Lijia Chen who have not been listed as authors of this article.

\section{References}

1. Boerma T, Ronsmans C, Melesse DY, Barros AJD, Barros FC, Juan L, et al. Global epidemiology of use of and disparities in caesarean sections. Lancet. 2018;392(10155):1341-8.

2. Liang J, Mu Y, Li X, Tang W, Wang Y, Liu Z, et al. Relaxation of the one child policy and trends in caesarean section rates and birth outcomes in China between 2012 and 2016: observational study of nearly seven million health facility births. Bmj. 2018;360:k817.

3. Li HT, Luo S, Trasande L, Hellerstein S, Kang C, Li JX, et al. Geographic Variations and Temporal Trends in Cesarean Delivery Rates in China, 2008-2014. Jama. 2017;317(1):69-76.

4. Cavallaro FL, Cresswell JA, Ronsmans C. Obstetricians' Opinions of the Optimal Caesarean Rate: A Global Survey. PLoS One. 2016;11(3):e0152779.

5. Betran AP, Temmerman M, Kingdon C, Mohiddin A, Opiyo N, Torloni MR, et al. Interventions to reduce unnecessary caesarean sections in healthy women and babies. Lancet. 2018;392(10155):1358-68.

6. Feng XL, Wang Y, An L, Ronsmans C. Cesarean section in the People's Republic of China: current perspectives. Int J Womens Health. 2014;6:59-74.

7. Zhang J, Liu Y, Meikle S, Zheng J, Sun W, Li Z. Cesarean delivery on maternal request in southeast China. Obstet Gynecol. 2008;111(5):1077-82. 
8. Long Q, Klemetti R, Wang Y, Tao F, Yan H, Hemminki E. High Caesarean section rate in rural China: is it related to health insurance (New Co-operative Medical Scheme)? Soc Sci Med. 2012;75(4):733-7.

9. Hellerstein S, Feldman S, Duan T. China's 50\% caesarean delivery rate: is it too high? Bjog. 2015;122(2):160-4.

10. Shearer EL. Cesarean section: medical benefits and costs. Soc Sci Med. 1993;37(10):1223-31.

11. Sandall J, Tribe RM, Avery L, Mola G, Visser GH, Homer CS, et al. Short-term and long-term effects of caesarean section on the health of women and children. Lancet. 2018;392(10155):1349-57.

12. Gibbons L, Belizan JM, Lauer JA, Betran AP, Merialdi M, Althabe F. Inequities in the use of cesarean section deliveries in the world. Am J Obstet Gynecol. 2012;206(4):331.e1-19.

13. Obstetrics group of the Obstetrics and Gynecology Branch of Chinese Medical Association. Guidelines for Promoting Cervical Maturity and Induction of Labor in Late Pregnancy. (2014). Chinese Journal of Obstetrics and Gynecology. 2014;49(12):881-5.

14. Fan CF, Zhang ZE, Ming L, Chen JH. A control study of COOK double balloon dilation and oxytocin for cervical ripening and induction of labor. Chongqing Medicine. 2012;41(36):3820-2.

15. Duro-Gomez J, Garrido-Oyarzun MF, Rodriguez-Marin AB, de la Torre Gonzalez AJ, Arjona-Berral JE, Castelo-Branco $\mathrm{C}$. What can we do to reduce the associated costs in induction of labour of intrauterine growth restriction foetuses at term? A cost-analysis study. Arch Gynecol Obstet. 2017;296(3):483-8.

16. Jozwiak M, Bloemenkamp KW, Kelly AJ, Mol BW, Irion O, Boulvain M. Mechanical methods for induction of labour. Cochrane Database Syst Rev. 2012(3):Cd001233.

17. Lim SE, Tan TL, Ng GYH, Tagore S, Kyaw EEP, Yeo GSH. Patient satisfaction with the cervical ripening balloon as a method for induction of labour: a randomised controlled trial. Singapore medical journal. 2018;59(8):419-24.

18. ACOG Practice Bulletin No. 107: Induction of labor. Obstet Gynecol. 2009;114(2 Pt 1):386 - 97.

19. Organization WH. WHO recommendations for induction of labour. 2018 [cited 2018 Accessed June 26]. Available from: http://apps.who.int/iris/bitstream/10665/44531/1/9789241501156_eng.pdf..

20. Leduc D, Biringer A, Lee L, Dy J. Induction of labour. Journal of obstetrics and gynaecology Canada: JOGC = Journal d'obstetrique. et gynecologie du Canada : JOGC. 2013;35(9):840-57.

21. Medical CCRBC. Balloon catheters and labor induction 2014 [cited 201416 May]. Available from: Cookmedical.com.

22. F PAAM, R O-S. - ZHMSM. Evaluating The Impact of Risk Factors on Birth Weight and Gestational Age: A. D - 101487941. (- 2008-076X (Print)):T - ppublish.

23. I MG. S, H M, F P, R S. - Postterm pregnancy. D - 101578773. (- 2032 - 0418 (Print)):T - ppublish.

24. SM N. DA L. - Predicting live birth, preterm delivery, and low birth weight in infants born from. D 101231360. (- 1549-1676 (Electronic)):T - epublish.

25. RL G, JF C, JD I. R R. - Epidemiology and causes of preterm birth. D - 2985213r. (- 1474-547X (Electronic)):T - ppublish. 
26. Wu LX, Yang B, Yang J, Xu XF. Analysis on the change of cesarean section rate in a central district of Shanghai before and after the universal two-child policy. Chinese Journal of Woman Child Health Research. 2020;31(03):327-32.

27. Xu L, Deng LL. The impact of the second-child policy on the outcome of nulliparous women and analysis of relevant factors. Chinese General Practice Nursing. 2017;15(26):3304-5.

28. Zhang JS, Cheng HD, Zhang YP, Zhang XH, Xu XM, Zhang L, et al. Investigation and analysis of cesarean section rate and indications in Shanghai. Chinese Journal of Practical Gynecology Obstetrics. 2019;35(03):325-9.

29. Han J, Qin JY. Clinical analysis of COOK cervical dilation balloon for induced labor in full-term pregnancy. Chinese Journal of Minimally Invasive Surgery. 2016;16(07):597-600.

30. Sayed Ahmed WA, Ibrahim ZM, Ashor OE, Mohamed ML, Ahmed MR, Elshahat AM. Use of the Foley catheter versus a double balloon cervical ripening catheter in pre-induction cervical ripening in postdate primigravidae. J Obstet Gynaecol Res. 2016;42(11):1489-94.

31. Mei-Dan E, Walfisch A, Suarez-Easton S, Hallak M. Comparison of two mechanical devices for cervical ripening: a prospective quasi-randomized trial. J Matern Fetal Neonatal Med. 2012;25(6):723-7.

32. Xing Y, Li N, Ji Q, Hong L, Wang X, Xing B. Double-balloon catheter compared with single-balloon catheter for induction of labor with a scarred uterus. Eur J Obstet Gynecol Reprod Biol. 2019;243:139-43.

33. Boulvain M, Kelly A, Lohse C, Stan C, Irion O. Mechanical methods for induction of labour. Cochrane Database Syst Rev. 2001(4):Cd001233.

34. Atad J, Hallak M, Ben-David Y, Auslender R, Abramovici H. Ripening and dilatation of the unfavourable cervix for induction of labour by a double balloon device: experience with 250 cases. $\mathrm{Br}$ J Obstet Gynaecol. 1997;104(1):29-32.

\section{Supplementary Files}

This is a list of supplementary files associated with this preprint. Click to download.

- completedSTROBEcohortchecklist.docx 